\title{
Freiwilligkeit in der erwachsenenpädagogischen Beratung
}

\author{
Henning Pätzold · Susanne Ulm
}

Eingegangen: 10. März 2015 / Angenommen: 10. Juli 2015 / Online publiziert: 14. August 2015

(C) Die Autor(en) 2015. Dieser Artikel ist auf Springerlink.com mit Open Access verfügbar.

Zusammenfassung In der erwachsenen- und sozialpädagogischen Theorie und Praxis wird Freiwilligkeit als ein wenn nicht unbedingt notwendiges, so doch zumindest wünschenswertes Merkmal von Beratung betrachtet. Freiwilligkeit sollte damit auch ein wesentlicher Bestandteil der Planung, Gestaltung und Reflexion von Beratungsprozessen sein. Also benötigen professionelle Beraterinnen und Berater die Möglichkeit, auf entsprechende Reflexionsvorlagen und Strukturierungsangebote zurückgreifen zu können. Innerhalb der einschlägigen Literatur zeigt sich jedoch ein wenig präzises Bild der Dimension Freiwilligkeit in Beratungskontexten. So häufig, wie sie genannt wird, so selten wird sie präzise definiert. In diesem Artikel wird daher der Versuch unternommen, tiefer auf die unterschiedlichen Merkmale und Abstufungen von Freiwilligkeit in Beratungssituationen einzugehen und diese sinnvoll zu strukturieren. Konkretisiert werden die Ergebnisse, indem am Beispiel der Standards des Nationalen Forums Beratung Bezüge zu pädagogischen und professionellen Wertorientierungen hergestellt und diskutiert werden. Damit wird aufgezeigt, wie ein erwachsenenpädagogischer Umgang mit Freiwilligkeit konstruktiv bearbeitet werden kann.

Schlüsselwörter Regulative Beratung $\cdot$ Beratungsethik $\cdot$ Beratungstheorie Erwachsenenpädagogik · Freiwilligkeit

Abstract In adult education as well as in social work voluntariness is regarded as a desirable - if not paramount - characteristic in the process of counselling. Therefore

Dr. S. Ulm $(\bowtie) \cdot$ Prof. Dr. H. Pätzold

Universität Koblenz-Landau, Campus Koblenz,

Universitätsstr. 1,

56070 Koblenz, Deutschland

E-Mail: susanneulm@uni-koblenz.de

Prof. Dr. H. Pätzold

E-Mail: paetzold@uni-koblenz.de 
it should also be a fundamental part in the planning, performing and reflection of consultation. Thus professional counsellors need appropriate reflection templates and models in this regard. However, the discussion lacks a precise concept of voluntariness in the process of counselling. While it is often demanded, there rarely exist concise definitions. In this article, therefore, an attempt is made to go deeper into the different features and nuances of voluntariness in counselling situations and to structure them in a sensible way. The results are concretised by relating the standards of the National Forum of Counselling to educational and professional values. Thus we want to show a means to deal with matters of voluntariness from an adult education perspective.

Keywords regulatory counselling · ethics in counselling · counselling theory · adult education $\cdot$ voluntariness

\section{Einleitung: Von der Bedeutung der Freiwilligkeit}

Beratung ist ein ebenso populärer wie variantenreich definierter Begriff. In dem $\mathrm{Maße}$, in dem er für unterschiedliche Handlungs- und Lebensbereiche erschlossen wird, werden entsprechend vielfältige Merkmale von Beratung formuliert und von den Beratenden eine große Spannbreite von Kompetenzen verlangt. Und doch herrscht quer durch die Diskussionsbeiträge aus Theorie und Praxis ein breiter Konsens, dass Freiwilligkeit ein wenn nicht notwendiges, so doch zumindest wünschenswertes Merkmal von Beratung sei. So definierten Schwarzer und Posse (1986) Beratung als eine „freiwillige, kurzfristige, oft nur situative, soziale Interaktion zwischen Ratsuchenden (Klienten) und Berater" (1986, S. 634). Auch Kranz setzt die „Freiwilligkeit der Teilnahme und die jederzeit gegebene Freiheit zum Abbruch" im Kontext von Beratung voraus (2009, S. 356 f.). Andere Autoren vertreten die Auffassung, dass Freiwilligkeit zwar eine wünschenswerte Voraussetzung von Beratung darstellt, jedoch auch (erst) innerhalb der Beratungssituation hergestellt werden kann. Sie vermitteln damit ein weiter gefasstes Verständnis des Begriffes (vgl. Schäfter 2010; Warschburger 2009; Thiersch 2004). Ausnahmen räumt auch das Nationale Forum Beratung ein, indem es Freiwilligkeit einerseits zu einem Definitionsmerkmal von Beratung macht und andererseits anerkennt, dass ebenso Kontexte möglich sind, ,in denen Beratung obligatorisch ist und ggf. Sanktionen nach sich ziehen kann" (nfb 2014, S. 7). Ob Freiwilligkeit nun verlangt oder nur empfohlen wird, in jedem Fall macht sie offenkundig ein wesentliches Merkmal von Beratung aus, das bei der Planung, Gestaltung und Reflexion von Beratungssituationen große Aufmerksamkeit verdient. So würde man erwarten, dass hierzu - ähnlich wie zu anderen Aspekten von Beratung - eine Vielfalt konzeptionell-begrifflicher und praktischer Interpretationen vorliegt. Denn professionelle Beratende sollten auf Reflexionsfolien zurückgreifen können, um die unterschiedlichen Gestaltungsmöglichkeiten von Freiwilligkeit in der Beratung wie auch die verschiedenen denkbaren Rahmenbedingungen differenziert einschätzen und nutzen zu können. Und Lehrende, die im größer werdenden Handlungsfeld der Ausbildung von Beraterinnen 
und Beratern tätig sind, sollten gleichfalls über entsprechende Interpretations- und Strukturierungsangebote verfügen.

Der Blick in die Literatur zeigt hier aber - anders als in Bezug auf andere zentrale Aspekte von Beratung - ein eher diffuses Bild. Die oben dargestellten Beispiele belegen bereits, dass schon die grundsätzliche Bedingung Freiwilligkeit von verschiedenen Autorinnen und Autoren im Feld der Beratung sehr unterschiedlich bewertet wird. Hinzu kommt, dass für unterschiedliche Gestaltungsmöglichkeiten auch sehr verschiedene Begriffe verwendet und schließlich auch unterschiedliche Begründungen herangezogen werden (s. u.). So wichtig Freiwilligkeit als Thema von Beratung eingeschätzt wird, so unpräzise erscheint also ihre Behandlung in weiten Teilen der einschlägigen Literatur. Dadurch besteht insbesondere die Gefahr, dass Zusammenhänge zwischen Unfreiwilligkeit in und Steuerung durch Beratung übersehen oder falsch gedeutet werden. Im Folgenden sollen deshalb zunächst Merkmale von Freiwilligkeit diskutiert werden, die für diesen Umstand als mitverantwortlich gesehen werden. Weiterhin werden bestehende, bisher wenig zusammenhängende Differenzierungen des Konzepts Freiwilligkeit diskutiert und strukturiert. Ziel ist es, die oben angesprochene Problematik zu bearbeiten und zu einer konsistenteren Beschreibung der Formen von Freiwilligkeit in der Beratung zu gelangen. Um diese für erwachsenenpädagogische Planung, Handlung und Reflexion zu erschließen, wird sie in Beziehung zu normativen Orientierungen der Erwachsenenpädagogik gesetzt. Anhand ausgewählter Beispiele wird abschließend darauf eingegangen, wie sich Standards des Beratungshandelns durch den Bezug auf derartige normative Orientierungen konkretisieren lassen und wo sie gegebenenfalls an Grenzen stoßen. Vor diesem Hintergrund kann eine Einschätzung zur Frage gegeben werden, ob und wie Bedingungen der Unfreiwilligkeit (insbesondere im Falle regulativer Beratung) in erwachsenenpädagogischem Beratungshandeln begegnet werden kann.

\section{Freiwilligkeit: Begriffe und Deutungen}

\subsection{Normative und instrumentelle Freiwilligkeit}

Freiwilligkeit erscheint auf den ersten Blick als klares Konzept. Tut jemand etwas freiwillig, so ist es Resultat einer freien, durch keinen äußeren Zwang beeinflussten Willensentscheidung. Auf den zweiten Blick stellen sich allerdings Fragen. Zunächst wäre aus philosophisch-anthropologischer Perspektive zu klären: Hat der Mensch überhaupt einen solchen freien Willen bzw. wie kann sich die Pädagogik zu dieser Hypothese verhalten (vgl. Giesinger 2006)? Diese Frage kann an dieser Stelle nicht erörtert werden und ist unseres Erachtens mit pädagogischen Mitteln auch nicht zu klären. In der normativen Bezugnahme der Pädagogik auf die Aufklärung wird eine solche Möglichkeit jedoch vorausgesetzt. Bildung begründet sich gerade daraus, dass der Mensch über die Fähigkeit verfügt, sich ,,seines Verstandes aus eigener Kraft zu bedienen“" (Kant) und also auch freie Willensentscheidungen zu treffen - Hügli beschreibt diesen Gedankengang als Grundlage der sogenannten „Autonomiepädagogik“ (1999, S. 40). Pädagogik hat somit die Aufgabe, den Menschen bei der Entwicklung dieser Fähigkeit zu unterstützen. Frühzeitig wurde in diesem Zusam- 
menhang das Dilemma der pädagogischen Antinomie angesprochen (vgl. Giesinger 2006, S. 47): Einen Menschen zur Freiheit zu führen, bedeutet eben, ihn zu führen und gleichzeitig davon auszugehen, dass er bei voller Entfaltung seines Potenzials keinerlei Führung bedarf.

Auch erwachsenenpädagogische Beratung bezieht sich auf diese normative Position. Geht man vom Potenzial zur Selbstbestimmung aus, so verbietet es sich, diese über ein unbedingt notwendiges Maß hinaus zu beschneiden. Unter dieser Voraussetzung wäre eine Verpflichtung zur Beratung schwer zu begründen. Denn selbst wenn die Autonomiepädagogik Pflicht nicht grundsätzlich ablehnt, ist sie zumindest in Bezug auf die Handlungsform Beratung wenig plausibel. So liefert die Aufklärung in der pädagogischen Tradition eine Begründung für Freiwilligkeit in der Beratung, an die in allgemeinerer Form auch der Professionalitätsdiskurs der Erwachsenenpädagogik (vgl. Gieseke 2009; Nittel 2000; Tietgens 1988) anschließt.

Ein historisch durchaus ebenfalls wirkmächtiger Gegenentwurf zur Autonomiepädagogik liegt in der „Kontrollpädagogik“ (Hügli 1999, S. 53). Sie knüpft nicht an die Aufklärung an, sondern einerseits an politisch-instrumentelle Vorstellungen von der Lenkung von Menschen durch soziale Einflussnahme, andererseits an das technische Konzept des Regelkreises. Hier wird Autonomie weder als Ziel noch als Voraussetzung angenommen. Dennoch kann es auch hier sinnvoll erscheinen, Freiwilligkeit anzustreben, wenn die Annahme besteht, dass ein Ziel unter der Bedingung von Freiwilligkeit leichter oder besser erreicht wird. So legen zahlreiche Befunde der pädagogischen Psychologie nahe, dass bestimmte Lernergebnisse eher zu erreichen sind, wenn die Situation von den Lernenden als selbstbestimmt und damit - zumindest weitgehend - frei von Zwängen wahrgenommen wird (vgl. u. a. Deci und Ryan 1993; Prenzel 1995; Konrad und Traub 2009). Freiwilligkeit ist in diesem Fall nicht normativ, sondern instrumentell begründet. Die grundsätzliche Position der Kontrollpädagogik wird in der Erwachsenenpädagogik oft durch außerprofessionelle, organisationale Vorgaben eingebracht, beispielsweise durch Vorgaben über zu erfüllende Teilnahmezahlen oder Ziele wie die Förderung von Beschäftigungsfähigkeit und Mobilität in der Weiterbildungsberatung (vgl. Käpplinger und Klein 2013, S. 332).

Ein wesentlicher Grund für die unklaren Positionen in der Diskussion um Freiwilligkeit und Beratung dürfte genau darin liegen, dass normative und instrumentelle Perspektiven sich überlagern. Wenn Freiwilligkeit gleichzeitig normativ und instrumentell begründet wird, trägt das aber nicht zur Klärung ihrer Bedeutung bei. Denn eine normative Begründung bedarf keiner instrumentellen Rechtfertigung: Wenn pädagogisches Handeln in einem bestimmten Zusammenhang Freiwilligkeit normativ voraussetzt, kann diese Voraussetzung nicht durch instrumentelle Überlegungen aufgehoben werden, sie muss aber auch nicht durch solche bekräftigt werden. Umgekehrt ist eine instrumentelle Begründung unabhängig von der normativen Position. Wenn sich gezeigt hat, dass eine bestimmte Maßnahme freiwillig besser gelingt, ist es nicht erheblich, ob Freiwilligkeit auch normativ angestrebt wird. Bei den folgenden Überlegungen werden deshalb instrumentelle und normative Begründungen jeweils getrennt voneinander berücksichtigt. 


\subsection{Abstufungen von Freiwilligkeit und Machtmittel}

Obwohl die Bearbeitung des Themas Freiwilligkeit in der Beratungsliteratur sehr unterschiedlich geschieht, lassen sich verschiedene Formen von Freiwilligkeit ausmachen, die im Folgenden systematisiert werden sollen. Zwischen den Polen völliger Freiwilligkeit und völligem Zwang liegt dabei eine Übergangszone. Völlige Freiwilligkeit liegt vor, wenn die Inanspruchnahme von Beratung vollkommen unabhängig von Sanktionen, der Gewährung von Vorteilen usw. ist. Ein Beispiel für derartige äußere Freiwilligkeit ist ein privat in Anspruch genommenes berufliches Coaching, bei dem beispielsweise eine Führungskraft individuell beratende Unterstützung in Anspruch nimmt, um berufliche Anforderungen besser, leichter oder angenehmer zu bewältigen. Völliger Zwang läge hingegen vor, wenn die Klientin oder der Klient verpflichtet ist, sowohl die Beratung in Anspruch zu nehmen als auch die Ergebnisse umzusetzen. Derartige Bedingungen finden sich unter anderem in totalen Institutionen. Aber auch die Mitwirkungspflichten im Rahmen des Sozialgesetzbuches (SGB I, $\S \S 60$ ff.) können zu Situationen führen, in denen Klienten, die Beratung nicht in Anspruch nehmen oder Ergebnisse nicht umsetzen, mit schwerwiegenden Sanktionen zu rechnen haben. Zwischen diesen Polen liegen verschiedene Abstufungen von Freiwilligkeit und Zwang, die je nach Beratungskontext auf unterschiedlichen Dimensionen ausgeprägt sein können: Diese betreffen die

- Interaktion, d. h. inwieweit die Klientin oder der Klient sich auf die Beratungsinteraktionen einlässt,

- Aufrechterhaltung der Teilnahme, d. h. in welchem Umfang die Klientin oder der Klient das Recht hat, die Beratung aus eigenem Entschluss abzubrechen und die

- Nutzung der Ergebnisse, d. h. ob und in welchem Umfang die Klientin oder der Klient verpflichtet ist, Beratungsergebnisse in Handlungen umzusetzen.

Des Weiteren lassen sich als mögliche Konsequenzen von Verweigerung oder NichtKooperation in Bezug auf verpflichtende Beratung unterschiedliche Formen von Machtmitteln unterscheiden. Sie begründen sich einerseits in der Person und andererseits in der Position des Beratenden und entsprechen strukturell denjenigen der Führungsethik (vgl. Tab. 1).

Jede dieser Ausprägungen von Macht kann je nach Beratungskontext unterschiedlich entfaltet werden. Beratende verfügen je nach Position über unterschiedlich stark ausgeprägte Amtsautorität. In offensichtlichen Zwangskontexten haben sie Bestrafungs- oder Belohnungsmacht inne. Bestrafungen können etwa in der Kürzung von Transferleistungen oder der Verhängung von Zwangsgeldern bestehen, Belohnungen

Tab. 1 Formen von Führungs-/Beratungsmacht (nach Weibler und Kuhn 2012, S. 20 f., erweitert)

\begin{tabular}{ll}
\hline Positionale Macht & Personale Macht \\
\hline Amtsautorität & Expertenmacht \\
Belohnungsmacht & Überzeugungsmacht \\
regulative Macht & charismatische Macht \\
Bestrafungsmacht & Identifikationsmacht \\
Informationsmacht & \\
\hline
\end{tabular}


in der Zugänglichmachung besonderer Leistungen. Ferner gibt es die Möglichkeit, dass ein (Rechts-)Anspruch auf bestimmte Leistungen an Beratung geknüpft wird (beispielsweise bei der Förderung von Weiterbildung). Da hier weder eine Belohnung noch eine Bestrafung im eigentlichen Sinne vorliegen und die Beratung zum verpflichtenden Bestandteil der gewährten Leistung wird, sprechen wir - anschließend an die Begrifflichkeit von Käpplinger und Klein (2013, S. 241) - von regulativer Macht (vgl. auch Gerst 2002, S. 99). In pädagogischen Kontexten ist diese mitunter nicht scharf von der Informationsmacht zu trennen, etwa dann, wenn die Inanspruchnahme von Leistungen es erforderlich macht, über Informationen zu verfügen, die typischerweise den Klienten nicht verfügbar sind (beispielsweise aufgrund mangelnder Kenntnisse des Rechtssystems bei vielen Klienten der Migrationsberatung). Positionale Macht wirkt sich häufig als Teilnahmeverpflichtung aus und kann alle anderen Verpflichtungstypen einschließen.

Personale Macht besteht demgegenüber in Situationen, wo Klientinnen oder Klienten den Beratenden als Experten, als kompetent, als überzeugend, als charismatisch oder als Identifikationsperson wahrnehmen. Entsprechend kann sie in der Regel erst wirksam werden, wenn eine Interaktionssituation zustande kommt. Dann kann personale Macht sowohl dazu beitragen, dass die Interaktionssituation aufrechterhalten wird, als auch dazu, dass die Ergebnisse umgesetzt werden. Der Kontext der Führungstheorien, aber auch die Tradition des Herrschaftsbegriffs bei Max Weber zeigen dabei, dass hier auch die Dimension der Freiwilligkeit berührt ist: Einer Person zu vertrauen und ihren Rat anzunehmen, weil sie beispielsweise als charismatisch wahrgenommen wird, setzt nicht zwangsläufig voraus, auch schon freiwillig die Beratung in Anspruch genommen zu haben. Personale Macht kann hier also Unfreiwilligkeit bei der Wahrnehmung von Beratungssituationen überdecken.

Freiwilligkeit wird in der Literatur, wie oben angesprochen, vielfach thematisiert, kaum jedoch in systematischer Weise. Deshalb besteht eine Vielfalt unterschiedlicher Begriffe (z. B. „Zwangsberatung“, Rohner 2013, S. 142 ff. oder „Binnenfreiwilligkeit“, Pätzold 2004, S. 106) nebeneinander, die Aspekte und Ausprägungen von Freiwilligkeit beschreiben. Im Folgenden soll das dadurch abgesteckte Spektrum von Möglichkeiten systematisiert und mit - teilweise eingeführten - Begriffen beschrieben werden. Die Systematik (vgl. Tab. 2) begründet sich einerseits aus den logischen Unterordnungsverhältnissen (die Freiheit, eine Beratung abzubrechen, beschreibt einen geringeren Freiheitsgrad als die, zu wählen, ob Beratung überhaupt in Anspruch genommen wird), andererseits aus der normativen Orientierung. Weiterhin wird auf die oben beschriebene Machtperspektive und die genannten unterschiedlichen Dimensionen (Interaktion, Aufrechterhaltung der Teilnahme und Nutzung der Ergebnisse) eingegangen.

\subsubsection{Zwangsberatung und Pflichtklientschaft}

Viele sozialpädagogische - und zunehmend auch erwachsenenbildnerische - Beratungssituationen sind dadurch gekennzeichnet, dass sie für die Beratenden mit anderen Tätigkeiten in Konkurrenz stehen, z. B. Kontrollieren, Verwalten etc., was in der Sozialpädagogik treffend mit dem Begriff des „doppelten Mandats von Hilfe und Kontrolle" (Böhnisch und Lösch 1973) charakterisiert wurde. So bewegen sich 
Tab. 2 Typen, Begründungen, Beispiele und Differenzierungen von Freiwilligkeit in der Beratung

\begin{tabular}{|c|c|c|c|}
\hline Typ & $\begin{array}{l}\text { Normative und instrumen- } \\
\text { telle Begründung }\end{array}$ & Beispiele & Weitere Differenzierungen \\
\hline $\begin{array}{l}\text { äußere } \\
\text { Freiwilligkeit }\end{array}$ & $\begin{array}{l}\text { von der Autonomiepädago- } \\
\text { gik gefordert } \\
\text { in einigen Beratungskonzep- } \\
\text { ten instrumentell gefordert, } \\
\text { insbesondere, wenn Be- } \\
\text { ratung als notwendig er- } \\
\text { achtet wird und sonst nicht } \\
\text { zustande käme }\end{array}$ & $\begin{array}{l}\text { in vielen Beratungs- } \\
\text { kontexten gegeben, ein- } \\
\text { geschränkt etwa durch } \\
\text { Pflichtklientschaft }\end{array}$ & $\begin{array}{l}\text { vor allem nach positionalen } \\
\text { Machtmitteln (insbesondere } \\
\text { Belohnung, Bestrafung, } \\
\text { regulative Macht und } \\
\text { Informationsmacht) }\end{array}$ \\
\hline $\begin{array}{l}\text { Binnenfrei- } \\
\text { willigkeit }\end{array}$ & $\begin{array}{l}\text { von der Autonomiepäda- } \\
\text { gogik gefordert, auch als } \\
\text { Gegengewicht zu äußeren } \\
\text { Verpflichtungskontexten } \\
\text { instrumentell oft gefordert } \\
\text { oder empfohlen }\end{array}$ & $\begin{array}{l}\text { Schwangerschaftskon- } \\
\text { fliktgesetz: Verpflich- } \\
\text { tung zur Beratung bei } \\
\text { gleichzeitiger Frei- } \\
\text { heit zur Interaktion } \\
\text { mit Verweis auf den } \\
\text { Beratungscharakter }\end{array}$ & $\begin{array}{l}\text { Ausmaß der Interaktionsfrei- } \\
\text { willigkeit von völlig freier } \\
\text { Wahl für oder gegen Interak- } \\
\text { tionen bis zur Verpflichtung } \\
\text { zu „Rahmenhandlungen“ der } \\
\text { Beratung wie Angabe der } \\
\text { Personalien }\end{array}$ \\
\hline $\begin{array}{l}\text { verpflichtende } \\
\text { Freiwilligkeit }\end{array}$ & $\begin{array}{l}\text { normativ für Autonomiepäd- } \\
\text { agogik akzeptabel } \\
\text { instrumentell vor allem } \\
\text { didaktisch begründet }\end{array}$ & $\begin{array}{l}\text { „Modellversuch Lern- } \\
\text { beratung“, Professiona- } \\
\text { lisierungskonzepte im } \\
\text { Bereich Beratung }\end{array}$ & $\begin{array}{l}\text { Ausmaß der Verpflichtung } \\
\text { (z. B. Umfang und Intensität } \\
\text { von wahrzunehmenden Bera- } \\
\text { tungen, Dokumentation usw.) }\end{array}$ \\
\hline $\begin{array}{l}\text { Abbruchfrei- } \\
\text { willigkeit }\end{array}$ & $\begin{array}{l}\text { von der Autonomiepädago- } \\
\text { gik gefordert } \\
\text { instrumentell eher „tech- } \\
\text { nisch begründet“ (i. S. einer } \\
\text { „Kündigungsklausel“) }\end{array}$ & $\begin{array}{l}\text { Kündigungsklauseln im } \\
\text { Rahmen von vertraglich } \\
\text { vereinbarten Bestandtei- } \\
\text { len einer Weiterbildung }\end{array}$ & $\begin{array}{l}\text { Ausmaß der Nachteile bei } \\
\text { Abbruch (z. B. Verlust von } \\
\text { Kursgebühren oder Ansprü- } \\
\text { chen wie dem auf eine Wie- } \\
\text { deraufnahme der Beratung) }\end{array}$ \\
\hline $\begin{array}{l}\text { Freiwilligkeit } \\
\text { im Umgang } \\
\text { mit den } \\
\text { Ergebnissen }\end{array}$ & $\begin{array}{l}\text { normativ über die Auto- } \\
\text { nomiepädagogik hinaus oft } \\
\text { gefordert } \\
\text { instrumentell mitunter } \\
\text { unterlaufen }\end{array}$ & $\begin{array}{l}\text { instrumentelle Einfluss- } \\
\text { nahme auf Klienten zur } \\
\text { Erfüllung dienstlicher } \\
\text { Vorgaben (Fallzahlen, } \\
\text { Budgeteinhaltung usw.) }\end{array}$ & $\begin{array}{l}\text { Reflexion des Einsatzes per- } \\
\text { soneller Macht, Herstellung } \\
\text { von Transparenz bezüglich der } \\
\text { eigenen personellen Macht- } \\
\text { mittel gegenüber den Klienten }\end{array}$ \\
\hline
\end{tabular}

Beratende hier häufig im Spannungsfeld zwischen den Interessen ihrer Klienten und den Kontrollinteressen öffentlicher Steuerungsinstanzen (vgl. Böhnisch und Lösch 1973, S. 27). Beratung kann daher auch die Freiwilligkeit der Teilnahme ausschließen. Eine solche „Zwangsberatung“ findet dann statt, wenn „Beratung nicht freiwillig, sondern unter Androhung von negativen Konsequenzen“ (Rohner 2013, S. 142) stattfindet, wobei diese Androhung nicht unbedingt offen erfolgen muss. Der Begriff der „Pflichtklientschaft“ (Laub 2008) geht ebenfalls auf einen Zwangskontext von Beratung ein, wenn Beratung behördliche oder gerichtliche Anordnungen zur Grundlage hat (vgl. Laub 2008, S. 25). Die Verpflichtung oder der Zwang zur Teilnahme an Beratung wurde hier vorab durch einen Rechtsakt hergestellt. Bernler und Johnsson (1997) benutzen den weiteren Begriff der ,erzwungenen Klientenschaft“ und Gröning (2011) merkt ferner dazu an, dass es

zum Regelwerk des Wohlfahrtsstaates (gehört), dass immer mehr Professionelle zwangsweise aufgesucht werden müssen, die Konsultation ist quasi vorgeschrieben. Nichtbefolgen bedeutet den Verlust von Ansprüchen. Damit ist der Autonomieanspruch des Klienten gebrochen oder doch zumindest vermindert (2011, S. 92). 
Diese institutionelle Einbindung bestimmt damit die Struktur des Zusammentreffens zwischen Klient und Berater nicht nur hinsichtlich der Freiwilligkeit, sondern auch im Hinblick auf Ort, Häufigkeit, Dauer der Treffen sowie der Zeitspanne der Zusammenarbeit (vgl. Schäfter 2010, S. 44 f.).

Erzwungene Klientschaft tritt typischerweise dort auf, wo ein (oft staatlicher) Akteur durch die Beratung ein bestimmtes Ergebnis sicherstellen möchte, beispielsweise maximale Anstrengungen eines Klienten beim Versuch des Wiedereintritts in den ersten Arbeitsmarkt. Ebenso kann sie dazu dienen, bestimmte Ereignisse zu verhindern oder zumindest frühzeitig zu erfahren, z. B. in der Bewährungshilfe (und mitunter wird es hier instrumentell als Vorteil angesehen, dass durch Pflichtklientschaft Beratung überhaupt erst zustande kommt, vgl. Laub 2008, S. 28 f.). Darüber hinaus kann sie aber auch dazu dienen, die individuelle Bearbeitung einer gesellschaftlich anerkannten Problemlage (Arbeitslosigkeit, Delinquenz, Krankheit usw.) zu strukturieren und kontinuierlich zu beeinflussen. Beratung wird dann im obigen Sinne regulativ eingesetzt, gewissermaßen ,,als ein Transmissionsriemen für das reibungslose Funktionieren des Programms““ (Laub 2008).

\subsubsection{Binnenfreiwilligkeit}

Auch wenn die Teilnahme an Beratung verpflichtend ist (beispielsweise im Zusammenhang mit Schwangerschaftskonflikten), gibt es unterschiedliche Möglichkeiten der Ausgestaltung der eigentlichen Beratungssituation. Mit dem Begriff der „Binnenfreiwilligkeit" (Pätzold 2004, S. 106) wird eine Situation beschrieben, in der die Beratung der äußeren Form nach zwar verpflichtend stattfindet, es also ein Zusammentreffen zwischen Berater und Klient gibt, der Klient aber nicht verpflichtet ist, sich auf ein eigentliches Beratungsgespräch einzulassen, sondern beispielsweise auch zu schweigen oder zu anderen Themen als dem Beratungsanlass zu sprechen. Bei der Binnenfreiwilligkeit ist damit insbesondere die Ebene der Interaktion angesprochen; sie kann in sich weiter abgestuft werden: So ist es zum Beispiel in der Schwangerschaftskonfliktberatung notwendig, dass die Klientin ihren Namen angibt, um die gesetzlich vorgeschriebene Bescheinigung (StGB, § 219) zu erhalten. Sie kann allerdings gegenüber der oder dem Beratenden anonym bleiben (SchKG, § 6). Die Beratung selbst ist im Gesetz einerseits inhaltlich beschrieben, andererseits stellt der Gesetzgeber fest: „Der Beratungscharakter schließt aus, dass die Gesprächs- und Mitwirkungsbereitschaft der schwangeren Frau erzwungen wird" (SchKG, § 5, Abs. 2). Es ist unklar, ob hierfür normative oder instrumentelle Gründe im Vordergrund stehen. Binnenfreiwilligkeit lässt sich allgemein aus beiden Perspektiven begründen. Aus normativer Sicht erscheint sie dort notwendig, wo ein äußerer Zwang der eigentlich gebotenen Teilnahmefreiwilligkeit entgegensteht. Aus instrumenteller Sicht erscheint sie häufig sinnvoll, um etwa defensive Tendenzen im Beratungsprozess (vgl. Faulstich et al. 2005, S. 24 ff.) zu vermeiden.

\subsubsection{Verpflichtende Freiwilligkeit}

Von Bedeutung ist weiterhin, unter welchen Bedingungen eine Klientin oder ein Klient die Beratung abbrechen kann. In instrumenteller Hinsicht kann es in erwachsenen- 
pädagogischen Angeboten sinnvoll oder sogar notwendig sein, eine Verbindlichkeit der Teilnahme - einschließlich der Teilnahme an Beratung - sicherzustellen. So wurde im „Modellversuch Lernberatung“ (Volk-von Bialy 1991) mit dem Begriff der „verpflichtenden Freiwilligkeit“ (Volk-von Bialy 1991, S. 46) der Umstand bezeichnet, dass die Adressaten zwar frei wählen können, ob sie ein erwachsenenpädagogisches Angebot wahrnehmen, sich mit der Entscheidung für die Teilnahme aber der verpflichtenden Beratung unterwerfen. Eine ähnliche Situation besteht etwa, wenn Teilnehmende einer Beratungsweiterbildung verpflichtet sind, innerhalb der Beratung auch selbst an (an sich freiwilligen) Supervisions- oder Beratungsangeboten teilzunehmen. Verpflichtende Freiwilligkeit kann in einem gewissen Maße auch die Verpflichtung zur Nutzung der Beratungsergebnisse umfassen, insbesondere wenn die Beratung als Teil einer Bildungsmaßnahme konzipiert ist. Werden beispielsweise im Rahmen der Beratung Aufgaben formuliert, die der Klient oder die Klientin bearbeiten kann, so kann dies mit Blick auf das Bildungsziel einer solchen Maßnahme verpflichtend geschehen. In der Regel besteht unter der Bedingung verpflichtender Freiwilligkeit allerdings weiterhin Abbruchfreiwilligkeit, die Möglichkeit also, die Beratungssituation (jederzeit) endgültig verlassen zu können (wenngleich hierbei natürlich sekundäre Nachteile wie z. B. der Verlust eventuell gezahlter Kursgebühren entstehen können).

\subsubsection{Freiwilligkeit im Umgang mit den Ergebnissen von Beratung}

Selbst wenn Beratung nicht gänzlich als freiwillig konzipiert wird, herrscht doch oft die Vorstellung vor, dass zumindest der Umgang mit den Ergebnissen freiwillig ist, d. h. Klienten in vollem Umfang selbst entscheiden können, ob sie bestimmte Ergebnisse oder auch Aufgabenvorschläge der Beratenden umsetzen, modifizieren oder gar nicht aufgreifen möchten. Eine äußere Verpflichtung zur Umsetzung von Beratungsergebnissen reicht sehr tief in die persönlichen Rechte der Klienten hinein und ist deshalb selten und dann vor allem in auch sonst durch Zwang charakterisierten Situationen zu finden (etwa im Rahmen der Bewährungshilfe). Sie schließt dann andere Freiwilligkeiten (etwa zur Teilnahme oder zur Interaktion) in der Regel ebenfalls aus.

Häufiger wird die Freiwilligkeit im Umgang mit den Ergebnissen durch weniger formalisierte Strukturen beeinträchtigt. Insbesondere spielen hier die Aspekte personaler Macht (vgl. Tab. 1) eine große Rolle. (Neo-)charismatische Ansätze von Führung etwa beruhen gerade darauf, dass über das Einbringen persönlicher Eigenschaften bei den Geführten das „Nacheifern“, „Loyalität und Akzeptanz“ oder gar „Gehorsam“ (Lang und Rybnikova 2014, S. 95) evoziert werden. Gleiches kann natürlich auch in Beratungssituationen geschehen. Wenn Beratende überdies vonseiten ihres Auftraggebers mit der Erfüllung bestimmter Zielvorgaben konfrontiert sind (etwa quantitative Erfolgsindikatoren in Bezug auf die Klienten) liegt es nahe, dass sie auch auf personale Machtmittel zurückgreifen, die eine formal gewährte Freiwilligkeit bei der Umsetzung von Ergebnissen unterlaufen. Auch hier findet sich also die mit dem Begriff „regulativ“ angesprochene Verbindung von Beratung mit Steuerung oder Führung. Tabelle 2 liefert eine abgestufte Systematik der Formen von Freiwilligkeit, Begründungen sowie Beispiele und weitere Differenzierungen. 


\section{Konsequenzen: Beratung, Freiwilligkeit und der Bezug zur Erwachsenenpädagogik}

Beratung ist einerseits eine Grundform pädagogischen Handelns (vgl. Klein 2010, S. 34). In der Sozialpädagogik stellt sie andererseits ein Konzept dar, dass sich in unterschiedlichen Methoden (hier könnte etwa an die verschiedenen Beratungsansätze gedacht werden) und daran anschließend einzelnen Techniken konkretisiert (vgl. Galuske 2013, S. 213 ff.). Es geht also um ein „Handlungsmodell“ (Galuske 2013, S. 172 ff.), welches Methoden und Techniken in einen sinnhaften Zusammenhang bringt. Erst ein solches Konzept erlaubt es, jene auf ihre Tauglichkeit zu bewerten. In der Erwachsenenpädagogik wird Beratung nicht in gleicher Weise als Konzept verstanden, sondern oft durch einzelne konkrete Merkmale wie den Adressatenbezug (vgl. Klein 2010, S. 34) oder Beratungsanlässe und ein darauf gründendes (bildungs-)karrierenbezogenes Verständnis (vgl. Schiersmann 2011, S. 748 f.) bestimmt. Daneben bestehen spezifische erwachsenenpädagogische Beratungskonzepte wie das der Lernberatung (vgl. Klein und Reutter 2005; Pätzold 2004), die allerdings auch häufig instrumentell gedacht sind, d. h. eine besondere Teilaufgabe von Erwachsenenbildung erfüllen sollen (vgl. kritisch hierzu Kossack 2006). Folgt man allerdings einem Verständnis von Erwachsenenbildung in der Tradition des Bildungsbegriffs und der Aufklärung, so liegt es nahe, an die normative Position der Autonomiepädagogik anzuschließen. Erwachsenenpädagogische Beratung soll dann zu Bildung beitragen und Freiwilligkeit wird in diesem Verständnis zur Bedingung von Beratung. Wo sie nicht gänzlich realisiert werden kann, ist es erwachsenenpädagogischer Beratung aufgegeben, ein möglichst hohes Maß an Freiwilligkeit aufrechtzuerhalten, etwa durch Varianten von Binnenfreiwilligkeit. Die Zwangskontexte, in denen - auch erwachsenenpädagogische - Beratung stattfindet, dürfen dabei jedoch nicht übersehen oder geleugnet werden. Erwachsenenpädagogische Beratungskompetenz bedarf deshalb insbesondere des souveränen Umgangs mit Gestaltungsfreiräumen innerhalb von konkreten, durch einen je unterschiedlichen Grad an Verpflichtung gekennzeichnete Beratungssituationen. Die Bedeutung von Freiwilligkeit soll deshalb abschließend entlang des ,Kompetenzprofil(s) für Beratende in Bildung, Beschäftigung und Beruf" (nfb 2014) betrachtet werden. Es unterscheidet vier Kompetenzgruppen (systemumfassende, prozessbezogene, organisationsbezogene und gesellschaftsbezogene Kompetenzen) mit insgesamt 17 einzelnen, durch Indikatoren operationalisierten Kompetenzen (vgl. nfb 2014, S. 10 ff.). Beispielhaft wird im Folgenden auf zwei Kompetenzen (,Professionelle Haltung und ethisches Verhalten“ sowie „Berücksichtigung gesellschaftlicher Ziele“) eingegangen.

\section{1 „Professionelle Haltung und ethisches Verhalten“}

Beratende, so fordert der erste Indikator $\mathrm{zu}$ dieser Kompetenz, „orientieren ihr Handeln und Verhalten an ethischen Prinzipien und machen diese transparent und erlebbar“ (nfb 2014, S. 12). Bewusst verzichtet die Darstellung darauf, „ethische Prinzipien“ zu konkretisieren, verweist jedoch auf „Ethikstandards der Beratung“ (nfb 2014). Man darf davon ausgehen, dass das Nationale Forum Beratung hier nicht annimmt, es gäbe allgemeine, in unterschiedlichsten Handlungsfeldern unstrit- 
tig anerkannte Ethikstandards der Beratung. Vielmehr wird auf diese Weise die Anwendbarkeit der Kompetenzstandards in unterschiedlichen Handlungsbereichen mit unterschiedlichen ethischen Standards gesichert. Für erwachsenenpädagogische Beratung bedeutet das konsequenterweise, Beratungshandeln an ethische Standards der Erwachsenenbildung zu knüpfen. Solche liegen nicht in geschlossener Form vor, es gibt aber ethische Orientierungen, die in der Erwachsenenbildung eine sehr umfassende Anerkennung finden (etwa der Anspruch der „Teilnehmerorientierung“, vgl. Schrader 2014, S. 19). Beratung hätte sich hier also nach Prinzipien wie dem der Teilnehmerorientierung zu richten, ist mithin den Bedarfen und Interessen der Teilnehmenden (und nicht beispielsweise einer Organisation) verpflichtet. Entsprechend müssen Beratende in der Erwachsenenbildung mit ethischen Orientierungen dieses Handlungsfeldes vertraut sein.

\section{2 „Berücksichtigen gesellschaftlicher Ziele“}

Das „Berücksichtigen gesellschaftlicher Ziele“ (nfb 2014, S. 18) zählt zu den gesellschaftsbezogenen Kompetenzen. Verlangt wird von den Beratenden u. a. die Auseinandersetzung mit ihrem gesellschaftlichen Auftrag. Einer der Kompetenzindikatoren stellt fest: Die Beratenden ,unterstützen die Ratsuchenden dabei, ihre individuellen Ziele in einen sinnvollen Zusammenhang mit gesellschaftlichen Zielvorstellungen zu bringen" (nfb 2014). Gemessen an diesem Ziel wäre Binnenfreiwilligkeit schon eine schwierige Rahmenbedingung, denn Unterstützung setzt bereits eine eigene Aktivität der Klienten voraus. Indem außerdem die Möglichkeit, dass es eventuell gar keinen sinnhaften Zusammenhang zwischen den individuellen und den gesellschaftlichen Zielvorstellungen geben könnte, nicht berücksichtigt wird, ist sogar schon die Freiwilligkeit bei der Annahme von Ergebnissen der Beratung tangiert: Die Akzeptanz der Möglichkeit der Harmonisierung individueller und kollektiver Interessen ist Voraussetzung für einen in dieser Richtung gelagerten Beratungsprozess. Offenkundig können also durch die „Berücksichtigung gesellschaftlicher Ziele“ regulative Mechanismen wirksam werden. Gleichwohl lässt auch diese Zielvorstellung praktische Handlungsspielräume offen, wenn nämlich die Beratenden bereit und in der Lage sind, fundamentale Widersprüche zwischen individuellen und gesellschaftlichen Zielen nicht nur zuzulassen, sondern ihrerseits beratend zu bearbeiten. Das erfordert die Fähigkeiten, einerseits die Zielsetzung des Beratungsangebots transparent zu machen (vgl. nfb 2014, S. 12), andererseits aber auch die eigene Rolle und die eigenen ethischen Reflexionsfolien zu kennen (vgl. nfb 2014).

\subsection{Beurteilung}

Die Beispiele zeigen, dass Kompetenzen, wie sie in den Standards des Nationalen Forums Beratung niedergelegt sind, geeignet sind, einen erwachsenenpädagogischen Umgang mit Freiwilligkeit konstruktiv zu bearbeiten. Während sie - entsprechend dem typischen Aufbau von Curricula und Kompetenzprofilen - allerdings additiv angelegt sind, ergeben sich aus dem Voranstehenden auch Überlegungen zu einer Hierarchie solcher Kompetenzen. Die „Berücksichtigung gesellschaftlicher Ziele“ etwa genügt nur dann den hier dargestellten Vorstellungen von maximaler Freiwilligkeit, 
wenn der Beratende bereit und in der Lage ist, vorher die Zielsetzungen der Beratung umfassend transparent zu machen und auch eigene Beweggründe in diesem Zusammenhang zu reflektieren und gegebenenfalls mit zu thematisieren. Andernfalls bleibt die Gefahr der Ausübung nicht-legitimer Machtmittel, insbesondere personeller Art, und damit das unreflektierte Ausführen eines an Beratung geknüpften regulativen Auftrags. Eben deshalb bedürfen die Kompetenzstandards der Rückbindung an ethische Standards innerhalb eines Handlungsfeldes. Diese erst definieren dann ein spezifisches Verhältnis zur Freiwilligkeit, indem sie - etwa im Beispiel der Teilnehmerorientierung - darauf verweisen, dass die Interessen der Teilnehmenden zu respektieren und zu berücksichtigen sind, auch wenn sie anderen Beratungszielen zuwider laufen sollten.

\section{Fazit}

Die Dimension Freiwilligkeit spielt innerhalb von erwachsenenpädagogischer Beratung eine gewichtige Rolle. Dennoch zeigt sich bei näherer Betrachtung in der Literatur eine komplexe, wenig strukturierte und unpräzise Darstellung des Begriffs. Um eine bessere Übersicht herzustellen und eine Orientierungshilfe zu geben, wurde in diesem Artikel der Versuch unternommen, die verschiedenen Typen, Begründungen und Differenzierungen von Freiwilligkeit im Beratungskontext herauszuarbeiten, an Beispielen $\mathrm{zu}$ veranschaulichen und systematisch $\mathrm{zu}$ ordnen. Hierdurch sollte auch insbesondere die Planung, Gestaltung und Reflexion von Beratungssituationen für die Beratenden erleichtert werden.

Auch erwachsenenpädagogische Professionalität lässt sich am Umgang mit der Dimension Freiwilligkeit im Kontext von Beratung ermessen. Am Beispiel der Standards des Nationalen Forums Beratung konnte dargestellt werden, dass sich ethische Reflexionshorizonte der Erwachsenenbildung konkret im Umgang mit Freiwilligkeit in der Beratung niederschlagen können und sollten. Diese Standards lassen hierfür Freiraum, indem sie zwar Bezüge zu ethischen und gesellschaftlichen Werten eröffnen, diese selbst aber nicht konkret benennen. Hier können berufsethische Vorstellungen ansetzen. Gerade im Umgang mit Freiwilligkeit zeigt sich dann, in welchem Umfang ein Berater oder eine Beraterin Spannungsverhältnisse zwischen der eigenen Professionalität und von außen (etwa durch Auftraggeber) einwirkenden Erwartungen austariert.

Beratung im Feld der Erwachsenenbildung kann also eine in ethischer Hinsicht herausfordernde Aufgabe sein. Insbesondere wenn Erwachsenenpädagoginnen und Erwachsenenpädagogen sich an Formen regulativer Beratung beteiligen, erfordert dies ein hohes Niveau der Reflexion eigener professioneller Ansprüche sowie die Fähigkeit, diesen im Beratungshandeln Geltung zu verschaffen. Es erscheint notwendig, die relevanten Rollen, Funktionen und Aufträge in der Beratung unverstellt sichtbar zu machen und Spannungsverhältnisse zwischen den Erwartungen und Zielen von Beratenen, Beratenden und den Auftrag gebenden Organisationen als solche erkennbar zu machen und ggf. zu thematisieren. Erwachsenenpädagogisch handelnde Beraterinnen und Berater haben nicht das Recht, bestehende Dimensionen von Freiwilligkeit (vgl. Tab. 2) einzuschränken, vielmehr ist es ihre Aufgabe, solche Spielräume offenzuhalten und wenn möglich schrittweise zu erweitern. 
Open Access Dieser Artikel wird unter der Creative Commons Namensnennung 4.0 International Lizenz (http://creativecommons.org/licenses/by/4.0/deed.de) veröffentlicht, welche die uneingeschränkte Nutzung, Verbreitung und Wiedergabe für beliebige Zwecke erlaubt, sofern Sie den/die ursprünglichen Autor(en) und die Quelle ordnungsgemäß nennen, einen Link zur Creative Commons Lizenz beifügen und angeben, ob Änderungen vorgenommen wurden.

\section{Literatur}

Bernler, G., \& Johnsson, L. (1997). Psychosoziale Arbeit. Eine praktische Theorie. Weinheim: Beltz.

Böhnisch, L., \& Lösch, H. (1973). Das Handlungsverständnis des Sozialarbeiters und seine institutionelle Determination. In H.-U. Otto \& S. Schneider (Hrsg.), Gesellschaftliche Perspektiven der Sozialarbeit (Bd. 2, S. 21-40). Neuwied: Luchterhand.

Deci, E. L., \& Ryan, R. M. (1993). Die Selbstbestimmungstheorie der Motivation und ihre Bedeutung für die Pädagogik. Zeitschrift für Pädagogik, 39, 223-238.

Faulstich, P., Forneck, H. J., Grell, P., Häßner, K., Knoll, J., \& Springer, A. (2005). Lernwiderstand-Lernumgebung - Lernberatung. Bielefeld: W. Bertelsmann.

Galuske, M. (2013). Methoden der Sozialen Arbeit: Eine Einführung (10. Aufl.). Weinheim: Beltz Juventa.

Gerst, D. (2002). Wandel betrieblicher Kontrollpraktiken im Lichte einer poststrukturalistischen Machtanalytik. SOFI-Mitteilungen, 30, 91-107.

Gieseke, W. (2009). Professionalisierung in der Erwachsenenbildung/Weiterbildung. In R. Tippelt \& A. von Hippel (Hrsg.), Handbuch Erwachsenenbildung/Weiterbildung (S. 385-403). Wiesbaden: VS Verlag für Sozialwissenschaften.

Giesinger, J. (2006). Erziehung der Gehirne. Willensfreiheit, Hirnforschung und Pädagogik. Zeitschrift für Erziehungswissenschaft, 9(1), 97-109.

Gröning, K. (2011). Pädagogische Beratung. Konzepte und Positionen (2. Aufl.). Wiesbaden: VS Verlag für Sozialwissenschaften.

Hügli, A. (1999). Philosophie und Pädagogik. Darmstadt: Wissenschaftliche Buchgesellschaft.

Käpplinger, B., \& Klein, R. (2013). Beratung bei Weiterbildungsgutscheinen. Zwischen Prüfung, Information und Entscheidungshilfe. In B. Käpplinger, R. Klein \& E. Haberzeth (Hrsg.), Weiterbildungsgutscheine: Wirkungen eines Finanzierungsmodells in vier europäischen Ländern (S. 327-346). Bielefeld: W. Bertelsmann.

Klein, R. (2010). Beratung. In R. Arnold, E. Nuissl \& S. Nolda (Hrsg.), Wörterbuch Erwachsenenbildung (2. Aufl, S. 34-35). Bad Heilbrunn: Klinkhardt.

Klein, R., \& Reutter, G. (Hrsg.). (2005). Die Lernberatungskonzeption. Baltmannsweiler: Schneider.

Konrad, K., \& Traub, S. (2009). Selbstgesteuertes Lernen. Grundwissen und Tipps für die Praxis (2., unveränd. Aufl.). Baltmannsweiler: Schneider.

Kossack, P. (2006). Lernen Beraten. Eine dekonstruktive Analyse des Diskurses zur Weiterbildung. Bielefeld: transcript.

Kranz, O. (2009). Interaktion und Organisationsberatung. Interaktionstheoretische Beiträge zu Profession, Organisation und Beratung. Wiesbaden: VS Verlag für Sozialwissenschaften.

Lang, R., \& Rybnikova, I. (2014). Aktuelle Führungstheorien und -konzepte. Wiesbaden: Springer Gabler.

Laub, M. (2008). Soziale Arbeit mit PflichtklientInnen. Sozialpsychiatrische Informationen, 38(4), $25-31$.

Nationales Forum Beratung in Bildung, Beruf und Beschäftigung (nfb) \& Forschungsgruppe Beratungsqualität am Institut für Bildungswissenschaft der Ruprecht-Karls-Universität Heidelberg (Hrsg.). (2014). Professionell beraten: Qualitätsstandards für die Beratung in Bildung, Beruf und Beschäftigung. Bielefeld: W. Bertelsmann.

Nittel, D. (2000). Von der Mission zur Profession. Stand und Perspektiven der Verberuflichung in der Erwachsenenbildung. Bielefeld: W. Bertelsmann.

Pätzold, H. (2004). Lernberatung und Erwachsenenbildung. Baltmannsweiler: Schneider.

Prenzel, M. (1995). Zum Lernen bewegen. Unterstützung von Lernmotivation durch Lehre. Blick in die Wissenschaft, 4(7), 58-66.

Rohner, B. (2013). Sozialarbeiterische Beratung in Gesellschaft. Wiesbaden: Springer VS.

Schäfter, C. (2010). Die Beratungsbeziehung in der sozialen Arbeit. Eine theoretische und empirische Annäherung. Wiesbaden: VS Verlag für Sozialwissenschaften. 
Schiersmann, C. (2011). Beratung im Kontext lebenslangen Lernens. In R. Tippelt \& A. von Hippel (Hrsg.), Handbuch Erwachsenenbildung/Weiterbildung (5. Aufl, S. 747-767). Wiesbaden: VS Verlag für Sozialwissenschaften.

Schrader, J. (2014). Ethik erwachsenenpädagogischen Handelns. Alltäglich gefordert, selten bedacht. Report. Zeitschrift für Weiterbildungsforschung, 1, 17-28.

Schwarzer, C., \& Posse, N. (1986). Beratung. In B. Weidenmann \& A. Krapp (Hrsg.), Pädagogische Psychologie (S. 631-666). München: Psychologie Verlags Union.

Thiersch, H. (2004). Sozialarbeit/Sozialpädagogik und Beratung. In F. Nestmann, F. Engel \& U. Sickendiek (Hrsg.), Das Handbuch der Beratung. Disziplinen und Zugänge (Bd. 1, S. 115-124). Tübingen: dgvt.

Tietgens, H. (1988). Professionalität für die Erwachsenenbildung. In W. Gieseke (Hrsg.), Professionalität und Professionalisierung (S. 28-75). Bad Heilbrunn: Klinkhardt.

Volk-von Bialy, H. (1991). Modellversuch Lernberatung. Konzept einer Fortbildung Lernberatung für das Berufsförderungswerk Hamburg, Bd. 1 von Modellversuch Lernberatung. Berlin: Bundesinstitut für Berufsbildung.

Warschburger, P. (Hrsg.). (2009). Beratungspsychologie. Heidelberg: Springer.

Weibler, J., \& Kuhn, T. (2012). Führungsethik in Organisationen. Stuttgart: Kohlhammer. 